\title{
Thermal burn injury associated with a forced-air warming device
}

\author{
Kyudon Chung, SangMook Lee, Sae-Cheol Oh, Jihye Choi, and Hyun-Sook Cho \\ Department of Anesthesiology and Pain Medicine, College of Medicine, The Catholic University of Korea, Seoul, Korea
}

Although forced-air warming systems are highly effective in maintaining or restoring normothermia in surgical patients, they pose burning hazards to patients. The Food and Drug Administration (FDA) has issued a warning that misuse of forced-air warming units without blankets to treat systemic hypothermia can result in a burn [1]. We present a case in which a 37-year-old female suffered a burn injury associated with misuse of a forced-air warming device.

A 37-year-old female was admitted to our hospital for arthroscopic left knee surgery. Her medical history revealed type 2 diabetes of 5 years' duration. The operation was uneventful for an hour and a half under epidural anesthesia. The patient was intravenously premedicated with $3 \mathrm{mg}$ of midazolam. Twenty $\mathrm{ml}$ of $0.75 \%$ ropivacaine mixed with 50 $\mu \mathrm{g}$ of fentanyl was used for the epidural anesthesia. There was no complication during the administration of the epidural anesthesia, and the blocking level was T10. After the operation was finished, the patient was transferred to the post-anesthesia care unit. Because the patient began complaining that she felt cold after the surgery, she was heated with a Bair Hugger warming device (Model 505; Arizant Healthcare, Inc., Eden Prairie, MN, USA). The nozzle of the device was not connected to the disposable baffles designed specifically for use with forced-air warming devices, and $43^{\circ} \mathrm{C}$ hot air was blown directly to the patient for 30 minutes. There was no specific complaint at the post-anesthesia care unit. Thirty minutes later, the patient was transferred to the ward. She claimed that she had noticed large blisters on her abdomen when she was at the ward after her operation. She also complained about the warming with the forced air-warming device at the post-anesthesia care unit. Upon examination, bullae and a $5 \times 10 \mathrm{~cm}$ burn-like skin lesion on the patient's left lower abdomen was documented (Fig. 1). After the bullectomy, 12 days of wound care was required.

In the operating room and the post-anesthesia care unit, we often use forced-air warming systems. Forced-air warming is the process of using heated air to warm patients through convection. A forced-air warming device comprises an electrical heat generator, a hose, and a blanket. Hot air generated by the electrical heater is transferred to the blanket via the hose. Burn injuries associated with forced-air warming systems are very rare when the device is used according to the manufacturer's instructions [2]. The device always has considerable risk of

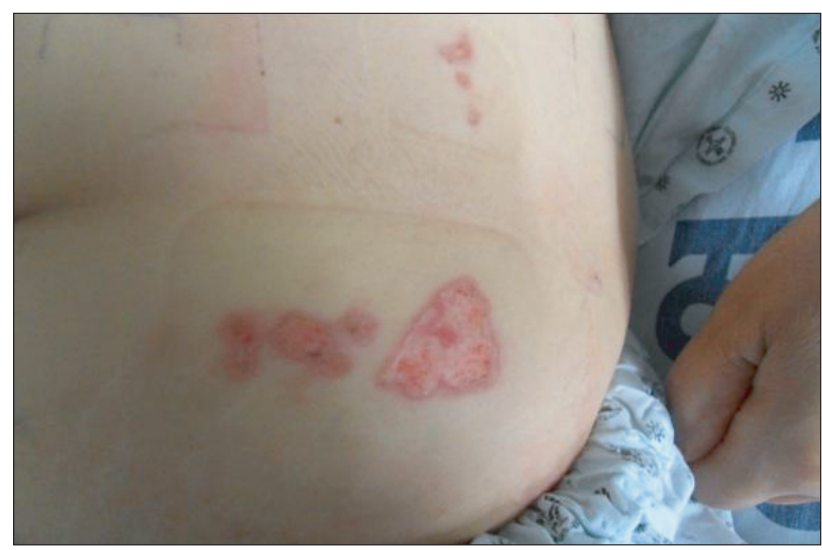

Fig. $1.5 \times 10 \mathrm{~cm}$ bullae and burn-like skin lesion on the patient's left lower abdomen.

Corresponding author: Hyun-Sook Cho, M.D., Department of Anesthesiology and Pain Medicine, College of Medicine, The Catholic University of Korea, 520-2, Daeheung-dong, Jung-gu, Daejeon 301-723, Korea. Tel: 82-42-220-9046, Fax: 82-42-242-3500, E-mail: rae700@hanmail.net (c) This is an open-access article distributed under the terms of the Creative Commons Attribution Non-Commercial License (http:// creativecommons.org/licenses/by-nc/3.0/), which permits unrestricted non-commercial use, distribution, and reproduction in any medium, provided the original work is properly cited. 
burn injury to patients, though. General misuse of the system involves detaching the hose from the blanket and blowing hot air directly onto the patient's skin. This practice is called "hosing". It heats a single spot on the skin directly for an extended time period. The heated air, dangerously concentrated and elevated at the hose nozzle, is being blown directly onto the patient's skin. Contact between the skin and the hose surface may also cause thermal injury. One of the device manufacturers has started a campaign in a Web site (http://www.stophosing.com) to inform clinicians about the risks associated with forced-air warming units without a blanket.

At first, we thought the light source tip of the arthroscope might have caused the burn injury. The light source of an arthroscope or its light cable tip causes skin burns. Burying the end of the light cable within the polyethylene drape produces a hole as big as the end of the light cable within 15 seconds [3]. During the operation, though, there was no smog, combustion, or melting hole in the polyethylene drape. Moreover, the skin injury caused by the light source tip usually appears as a small, round burn. In this case, the burn can be associated with a forced-air warming unit.

Uzun et al. [4] reported a case of a third-degree burn injury associated with misuse of a forced-air warming device. Hot air $\left(40-43^{\circ} \mathrm{C}\right)$ was blown directly on the patient's leg for 2 hours. In our case, the patient was heated only for 30 minutes at the postanesthesia care unit, but the nozzle of the forced-air warming device was not connected to the disposable baffles designed specifically for use with forced-air warming devices. This could be one of the circumstances that precipitated the patient's burn.
The disposable baffles designed specifically for use with forced-air warming devices are essential in dissipating the tremendous heat delivered by the device. Although a few cases, including a severe burn injury of the lower extremities, have been reported [5], not everyone knows the dangers of "hosing" [4].

Also, a sedated or anesthetized patient is likely to be unable to withdraw or communicate discomfort, which would prevent a burn.

Thus, forced-air warming units should always be used with a blanket, in accordance with the manufacturers' instructions, to protect patients from thermal injury. Moreover, we should pay attention to the device temperature, the attachment of the hose to the blanket, and the skin of the patient while using a forcedair warming system.

\section{References}

1. Marders J. FDA Encourages the reporting of medical device adverse events: free-hosing hazards. APSF Newslett 2002; 17: 42.

2. Bräuer A, Quintel M. Forced-air warming: technology, physical background and practical aspects. Curr Opin Anaesthesiol 2009; 22 : 769-74.

3. Smith LP, Roy S. Fire/burn risk with electrosurgical devices and endoscopy fiberoptic cables. Am J Otolaryngol 2008; 29: 171-6.

4. Uzun G, Mutluoglu M, Evinc R, Ozdemir Y, Sen H. Severe burn injury associated with misuse of forced-air warming device. J Anesth 2010; 24: 980-1.

5. Augustine S. Misuse of forced-air warming devices causes burns. APSF Newslett 2002; 17: 313. 